\title{
A Novel Tendon-Driven Soft Actuator with Self-Pumping Property
}

\author{
Tao Ren, ${ }^{1, *}$ Yingtian $\mathrm{Li}^{2,{ }^{2}}$ Menghong $\mathrm{Xu},{ }^{2}$ Yunquan $\mathrm{Li}^{2}{ }^{2}$ Caihua Xiong, ${ }^{3}$ and Yonghua $\mathrm{Chen}^{2}$
}

\begin{abstract}
Soft actuators and robotics have been widely researched in recent years mainly due to their compliance to environments and safe interaction with humans. However, the need of tether and low energy efficiency of such actuators/robots has limited their practical applications. This article presents a novel tendon-driven soft actuator concept that has the property of self-pumping, called soft self-pumping actuator (SSPA) in this research. A SSPA is designed by assembling two soft pneumatic actuators (SPAs) face-to-face, whose air chambers are connected by two check valves. Actuation of the SSPA is achieved by tendons that allows precise and untethered control compared with traditional SPAs. The two chambers in the proposed actuators are precharged with air to a desired pressure to enlarge self-stiffness and to facilitate bending. When actuated, one chamber will be compressed and serve as a pump to inject its air into the other chamber, resulting in further bending of the actuator. The airflow involves energy transmission to help the intended actuation, thus improving energy efficiency. In experimental studies, differential chamber air pressure is found to reduce the force in initiating actuator bending. Experimental results have also shown that energy efficiency increase of up to $45 \%$ has been achieved compared with the same design but without air transmission. We believe that the proposed concept could lead to more novel designs of controllable and energy saving soft robots.
\end{abstract}

Keywords: soft actuators, self-pumping, energy efficiency, tendon-driven robots, soft robots

\section{Introduction}

Q оғт Rовотісs is a buzzword in recent robotic researches. $\checkmark$ Researchers in this field have mainly focused on investigating mechanisms of bioinspired robots, ${ }^{1,2}$ developing and applying novel functional materials for soft robot actuations, ${ }^{3,4}$ proposing novel designs to accomplish complex tasks, ${ }^{5,6}$ and untethering and modularizing soft robotic designs. ${ }^{7-9}$ Even though important, the issue of low energy efficiency of soft robotics, in particular soft pneumatic actuators (SPAs), has seldom been addressed. Kofod et al. have reported a method to minimize energy consumption for filmbased actuators. ${ }^{10}$ High-efficiency actuation approach ${ }^{11}$ and novel stimulation means ${ }^{12,13}$ are also reported for soft robots based on different actuation principles. However, the issue of energy inefficiency ${ }^{14}$ is still a great challenge in the field of SPA-based soft robotics. For example, the energy efficiency of soft mobile robots is surprisingly low, mostly $<0.1 \%$, making them unrealistic for practical applications. ${ }^{15,16}$

\section{Limits and research gap \\ to pneumatic/tendon-driven actuators}

Pneumatic actuation is one of the most common means to power soft robotics or actuators. ${ }^{17}$ These SPAs are normally tethered to a bulky compressor/pump and complicated pneumatic valve control systems. The stiffness of the actuators can be tuned by modulating the pressure of air or liquid. However, to further expand the applications of SPA, it is desirable to get rid of the pneumatic pump and valve control system, and at the same time allow for more accurate bending and motion control. ${ }^{18}$ Tendon-driven soft actuators (TSAs) are another common type of soft actuators that often drive robotic joint motion through a pulley system. ${ }^{19,20}$ Compared

\footnotetext{
${ }^{1}$ Robotics Research Center, Xihua University, Chengdu, China.

${ }^{2}$ The Department of Mechanical Engineering, The University of Hong Kong, Hong Kong, China.

${ }^{3}$ State Key Laboratory of Digital Manufacturing Equipment and Technology, School of Mechanical Science and Engineering, Wuhan, China.

*These authors contributed equally to this work.
} 
with SPA, TSA is better in precision motion control and stiffness modulation. ${ }^{21}$ In the proposed soft self-pumping actuator (SSPA) in this research, air chambers are precharged with compressed air. Owing to the light weight, small size, and flexible routing of TSA, it can be easily embedded to a traditional SPA design, thus inspiring the concept presented in this research. Controlled tendon contraction will cause the proposed SSPA to bend, as well as airflow between the chambers, thus eliminating the need of pumps and pneumatic control systems for the operation of the proposed actuators. Furthermore, by contracting the tendons simultaneously, the stiffness of SSPAs can be controlled. Since there are no sudden air on/off operations, the SSPAs are also subjected to less vibration. ${ }^{22,23}$

Unlike SPA, the proposed actuator can be more accurately controlled. Compared with TSA, the stiffness of the SSPA is determined mainly by its structural design, material, and initial precharged air pressure, not solely by the tension of the tendons as in the case of TSA, even though the tension of the tendons does contribute a bit to the stiffness modulation of the SSPA. Precharged SPA with a single air chamber controlled by tendon actuation was reported previously. ${ }^{23}$ However, the tendons have to resist a large elastic force of the actuator, making it energy inefficient.

\section{Design concepts}

The proposed SPA with self-pumping property, abbreviated as SSPA, is inspired by the weighing scale as shown in Figure 1. In Figure 1A, the weighing scale is at its equilibrium. To break the equilibrium, only a very small perturbation force $F_{1}$ is needed to rotate the weighing scale counter-clockwise as shown in Figure 1B. The unbalance of the weighing scale will be accelerated if a portion of weight B slides to weight A as shown in Figure 1C. That is, weight B utilizes part of its own weight to further the unbalance, thus only a small force $F_{2}$ is needed to keep the unbalance. Now the weights in this concept are substituted by compressed air chambers, and air can flow between chambers to enhance the intended motion, thus only a smaller perturbation force $F_{2}$ is needed to keep the unbalance. This phenomenon is called self-pumping in this research.

Based on the mentioned concept, the proposed SSPA is designed with two identical traditional SPAs (Fig. 2A). Each SPA is precharged with compressed air. When the two precharged SPAs are put face to face, they reach an equilibrium if their precharged air pressures are the same (the left SPA tries to bend to the right and the right SPA tries to bend to the left). If airflow between the two chambers is not allowed as shown in Figure 2B, we call this type of design buttress SPA
(BSPA). Otherwise, we call it SSPA as shown in Figure 2C. In an SSPA, air chambers are connected by two check valves, whose allowable flow directions are opposite. Before use, the chambers are precharged with compressive air to a pressure level as required, and then sealed as in the case of a basketball or bike wheel. The check valve allows the chambers to be equally or differentially charged (the cracking pressure of the two check valves can be different).

\section{Properties of the proposed actuator}

When the chambers are equally precharged to the same air pressure, the actuator is at an equilibrium state, the same as the weighing scale shown in Figure 1A. In the case of a BSPA as shown in Figure 2B, the actuator has no self-pumping effect because compressed air cannot flow between the two chambers. A force $F_{1}$ is needed to offset the elastic force of the charged actuator before it can break the equilibrium and initiate the bending process (Fig. 2D). However, if there is a differential pressure $\Delta P$ between the two chambers as shown in Figure 2C, then only a smaller force $F_{2}$ is needed to initiate the bending process (Fig. 2E) because the differential pressure between chambers has already offset the self-stiffness of the precharged actuator. Therefore, the proposed actuator can save energy in actuator bending.

Another property of the proposed SSPA is the airflow between the two chambers, resulting in a self-pumping effect. For the BSPA as shown in Figure 2B, under an external force $F_{1}$, the actuator will bend toward one end as shown in Figure 2D. Since there is no airflow between the two chambers, the compression of chamber A works like a compressed spring and chamber B works similar to a tensile spring. Therefore, there is a rapidly increasing internal force resisting the bending. To reduce the buildup of resistant force in chamber A, a pressure balance channel (two small tubes and two check valves) is built between chambers A and B as shown in Figure 2C. Now, under a bending process as shown in Figure 2E, the volume of chamber $\mathrm{A}$ is decreased but its air pressure will almost keep the same as the air is pumped to chamber B through the check valve. Therefore, the check valve system can help to ease the "compressed chamber A" and pump more air to chamber B. Pumping more air into chamber B will help the actuator's bending process. This is why the proposed actuator is called self-pumping SPA.

Therefore, the major contributions of this research are summarized as follows: (1) A novel TSA with self-pumping property is proposed. (2) The differential pressure between the two chambers of the actuator can offset the stiffness of the actuator and help initiate the bending motion. (3) The transmission of pneumatic energy from one air chamber to
A
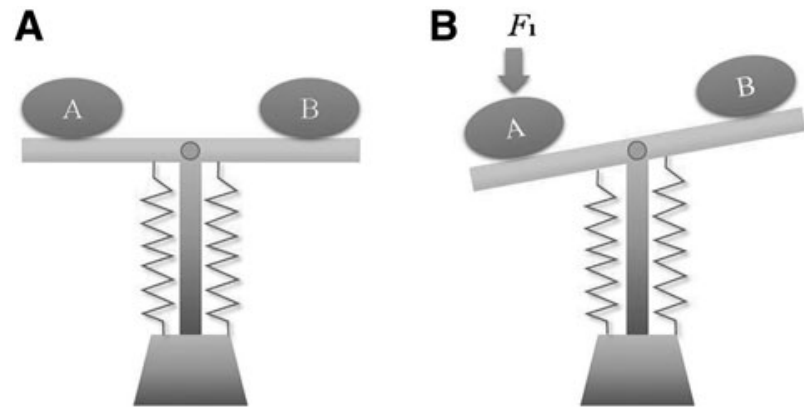

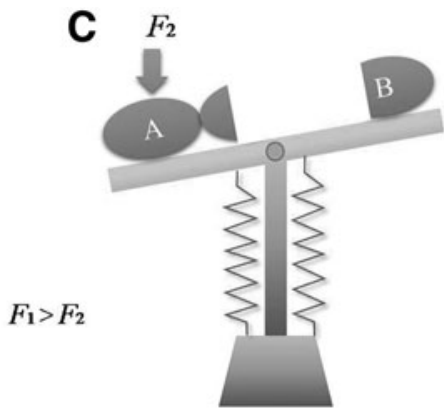

FIG. 1. Concept inspired by a weighing scale: (A) Weighing scale at equilibrium. (B) To break the equilibrium, a force $F_{1}$ is needed. (C) The unbalance can be accelerated if part of weight B is transferred to weight A. 
A

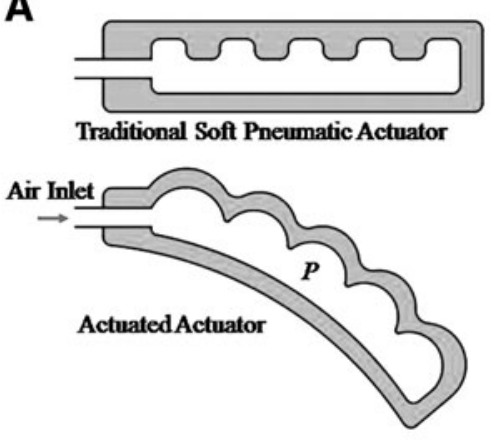

B

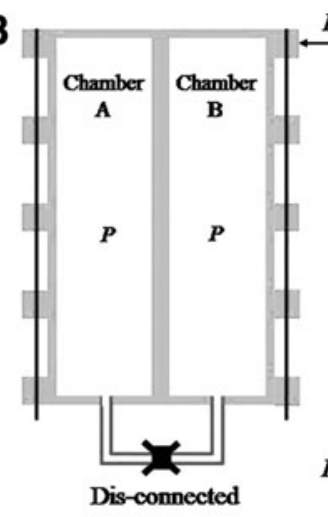

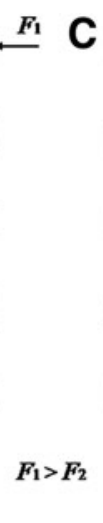
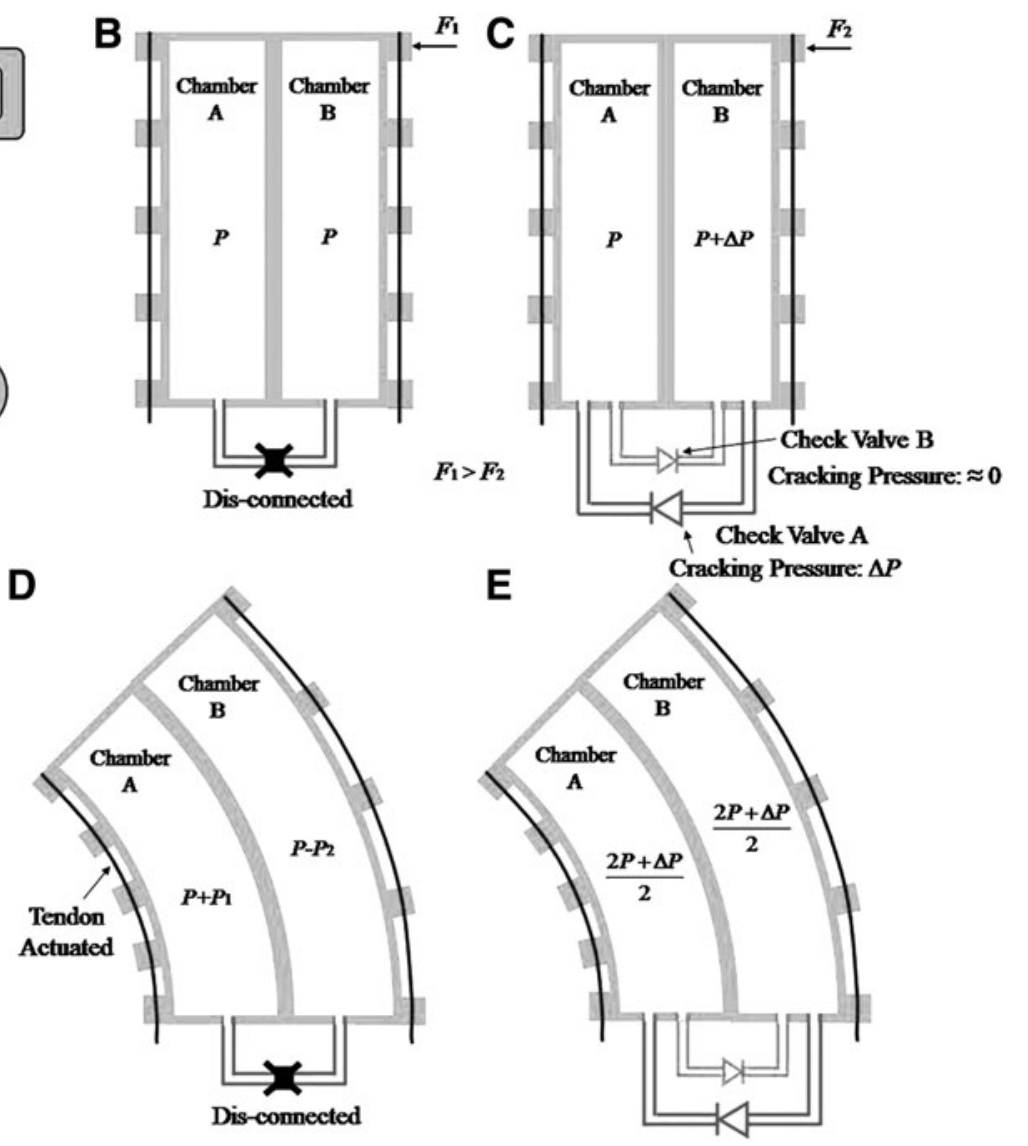

FIG. 2. The proposed SSPA design concept: (A) Traditional SPA, and its bending under compressed air $P$. (B) Two traditional SPAs put face to face (we define it as BSPA) with prepressurized pressure $\mathrm{P}$ in each chamber, a small force $F_{1}$ can break the equilibrium and initiate the bending. (C) Two air chambers connected by two check valves (we define it as SSPA) of different cracking pressures, giving a differential pressure $\Delta P$, an even smaller force $F_{2}$ is needed to bend the actuator. (D) Pull tendon to actuate the BSPA and the chamber pressure will change, but no air transfer. (E) Actuate the SSPA and the pressures of the two chambers will be balanced. SPA, soft pneumatic actuator; BSPA, buttress SPA; SSPA, soft self-pumping actuator.

another during bending can increase energy efficiency. (4) The proposed SSPA is simple and its operation is untethered. There is no need for a bulky pneumatic system while in operation.

\section{Modeling}

A key point of the proposed SSPA is the change of both volume and pressure of the actuator chambers during bending. The following provides a theoretic modeling of such changes. The actuation force is provided by a tendon drive as shown in Figure 3A in red.

To simplify the modeling, we make two hypotheses. (1) The total volume of chamber A plus chamber B is a constant during bending operation; (2) chamber $\mathrm{A}$ is the compressed chamber, and chamber B is the expanded chamber as shown in Figure 3B.

When the bending angle of the actuator is $\alpha$, the volume of the compressed chamber is $V_{A}$, and the expanded chamber is $V_{B}$. Let the initial volume of both chambers be $V_{0}$. We have

$$
V_{0}=\pi r^{2} l_{0} / 2
$$

where $l_{O}$ is the initial length of the actuator. Based on the geometrical relationship, the radius of the neutrosphere (Fig. 3B) is expressed as

$$
\rho=l_{0} / 2 \alpha
$$

Assume that there is no circumstantial strain, the circumstantial cross-section of the two chambers is two semicircles. Therefore, the volumes of the two chambers can be calculated through integration as

$$
\begin{aligned}
& V_{A}=(2 \alpha / 2 \pi) \int_{-\pi / 2}^{\pi / 2} \pi \rho^{2}-\pi\left(\rho-\sqrt{r^{2}-(r \sin \theta)^{2}}\right)^{2} d \theta \\
& V_{B}=(2 \alpha / 2 \pi) \int_{-\pi / 2}^{\pi / 2} \pi\left(\rho+\sqrt{\left(r^{2}-(r \sin \theta)^{2}\right.}\right)^{2}-\pi \rho^{2} d \theta
\end{aligned}
$$

After simplifying Eq. (3) and combining with Eqs. (1) and (2), the volume for each chamber at a given bending angle $\alpha$ is

$$
\begin{aligned}
& V_{A}=V_{0}-r^{3} \alpha \\
& V_{B}=V_{0}+r^{3} \alpha
\end{aligned}
$$

Now, we discuss the differences between the BSPA and the SSPA. For the BSPA, no air transfer will happen during bending. Thus, the pressure at the two chambers will be different in a bending operation. Based on the ideal gas law 
A

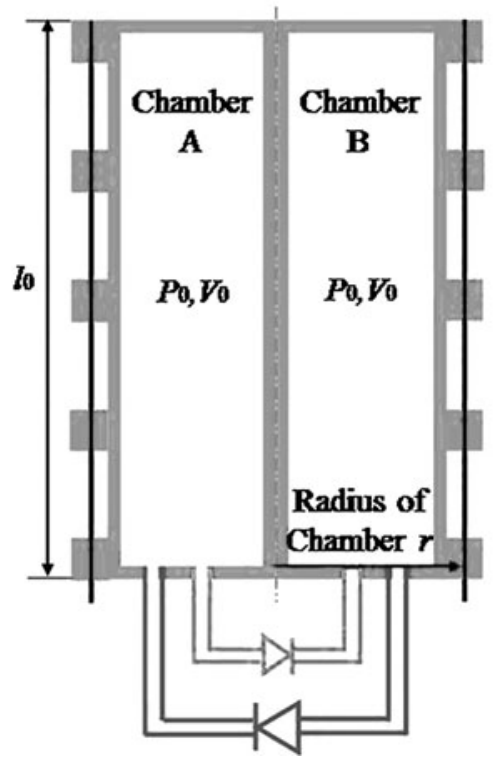

B

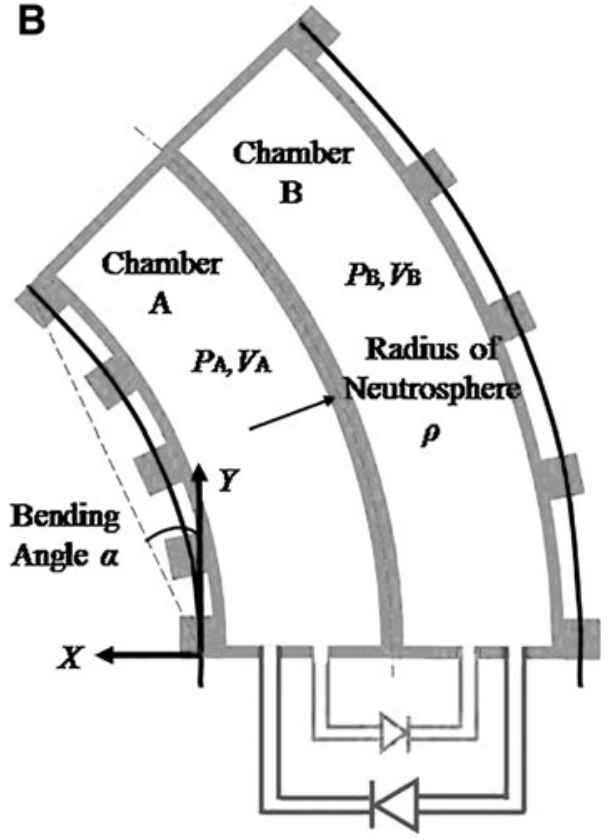

FIG. 3. Design modeling. (A) Initial state. (B) A bending state.
$P V=n R T$, the corresponding pressures inside the two chambers at a given bending angle $\alpha$ are expressed as

$$
\begin{aligned}
& P_{A}^{\prime}=P_{0} V_{0} /\left(V_{0}-r^{3} \alpha\right) \\
& P_{B}^{\prime}=P_{0} V_{0} /\left(V_{0}+r^{3} \alpha\right)
\end{aligned} .
$$

For the proposed SSPA, the two chambers are connected through a pressure balance channel. Assume there is no pressure difference at the initial state, the air pressure for both chambers at any bending angle $\alpha$ equals to the initial pressure and is expressed as

$$
P_{A}=P_{B}=P_{0}
$$

When design parameters of the actuator are known, the volume changes and pressure changes can be calculated based on Eq. (4). Figure 4 is a concept chart showing the volume and pressure changes against bending angle of the prototype used in this article. For both the BSPA and the SSPA, as shown in Figure 4A, the expanded chamber will increase its volume while the compressed chamber will decrease its volume. However, the chamber pressure changes in the BSPA and the SSPA are different. As shown in Figure 4B, the pressure of the expanded chamber of the BSPA decreases, whereas that of the compressed chamber increases. Because the pressure of the compressed chamber increases as the bending angle increases, its resistance to bending also increases. For the proposed SSPA, the pressure of both chambers remains the same value $P_{0}$. The pressure of the compressed chamber $\mathrm{A}$ is not increased even though bending angle increases. This is because part of its air is pumped into the expanded chamber B. Pumping of air into the expanded chamber B helps chamber B to further expand, just like the case of a normal SPA.
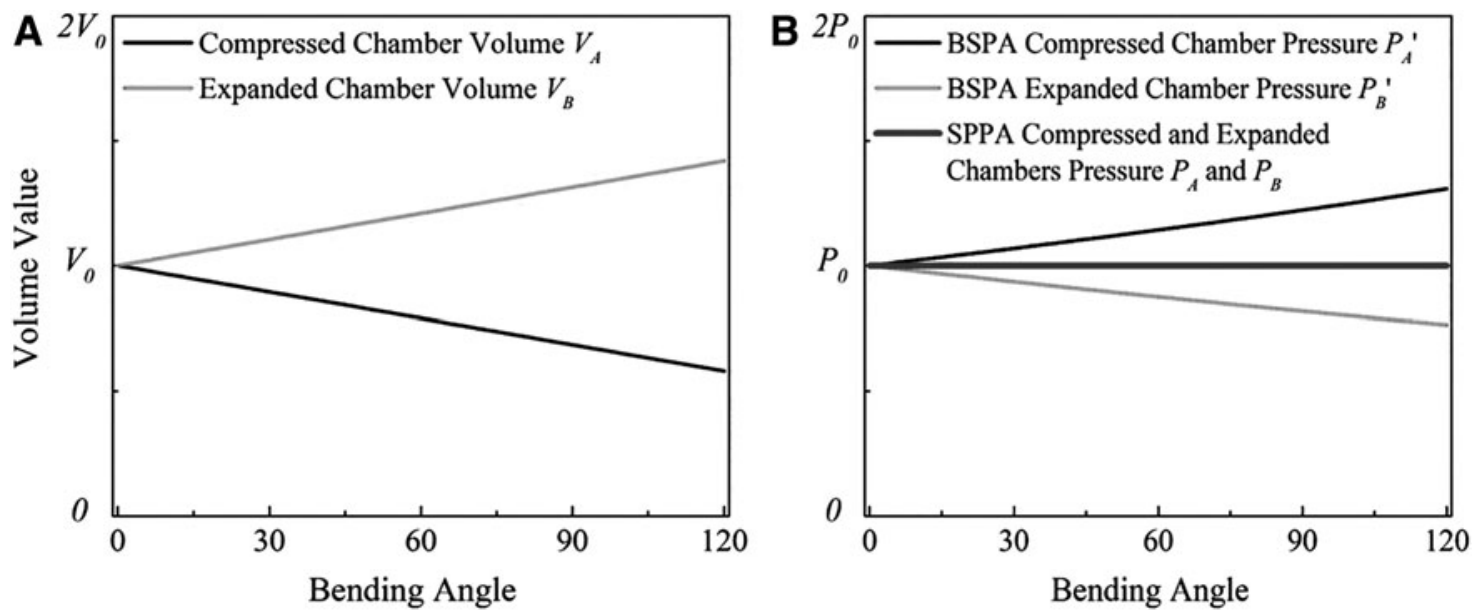

FIG. 4. Air chamber volume and pressure change against bending angle. (A) Chamber volume change against bending angle (same for both BSPA and SSPA). (B) Chamber pressure change against bending angle. 
A

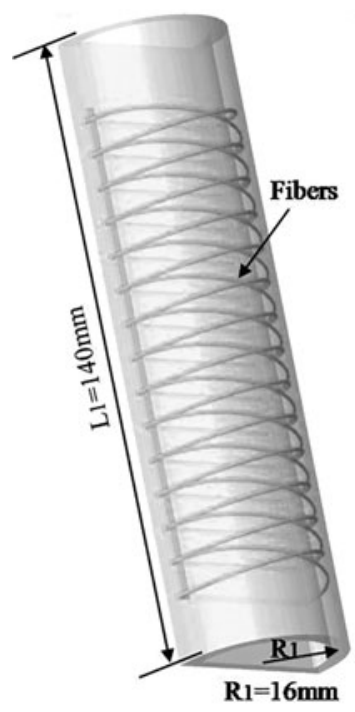

B

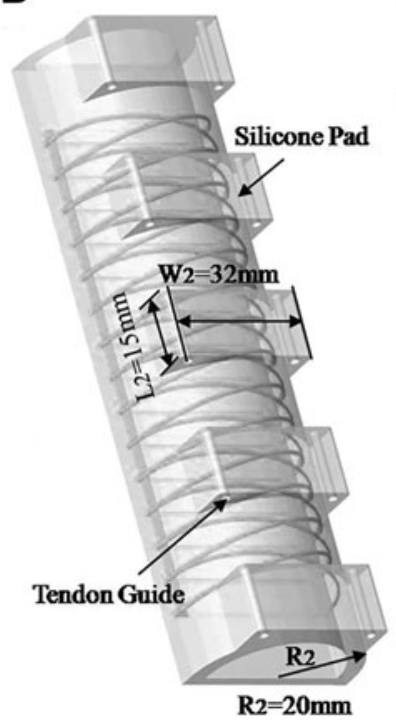

C

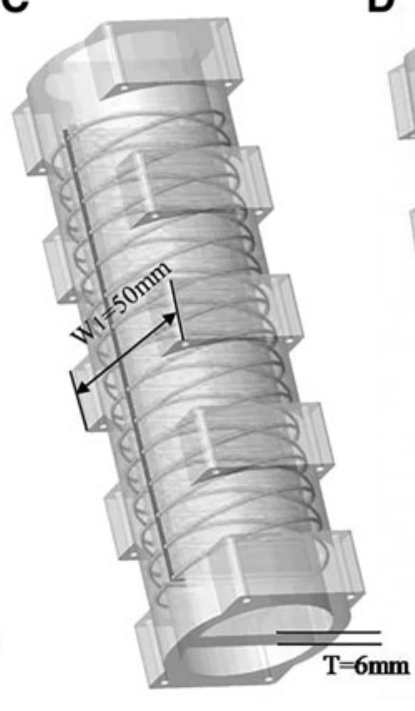

D

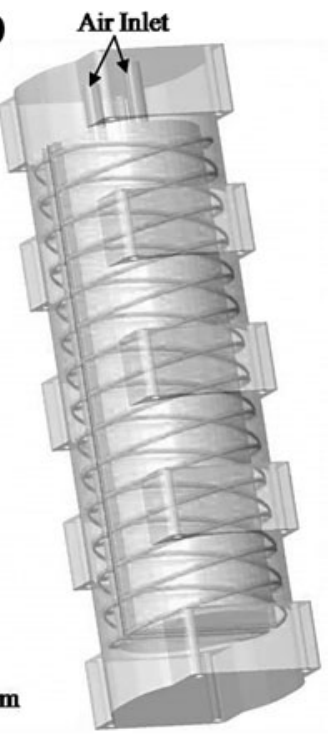

FIG. 5. A prototype SSPA design. (A) A traditional braided SPA with strain-limited layer. (B) Single chamber actuator molded with silicone pads and tendon guide channels. (C) Two actuators molded face to face. (D) Assemble the end cap and the air inlet valves.

\section{Actuator Design and Prototyping}

Figure 5 shows the three-dimensional (3D) schematic drawing with major parameters of a prototype SSPA. The main body of the actuator comprises three parts: the main body, tendon design, and the accessories. The main body of the SSPA is designed based on a slightly modified model of the widely studied classic fiber-reinforced SPA. ${ }^{24}$ The two chambers are identical having $140 \mathrm{~mm}$ in length. The crosssection of the chamber is a semicircle whose inner and outer radii are 16 and $20 \mathrm{~mm}$, respectively. The soft actuator is cast by silicone rubber with a hardness of 10D (from Shenzhen Yixing Chemical Co., Ltd.).

In this article, we have developed a robotic gripper to demonstrate the practicability of the proposed actuator as shown in Figure 6A. It shows the complete assembly of
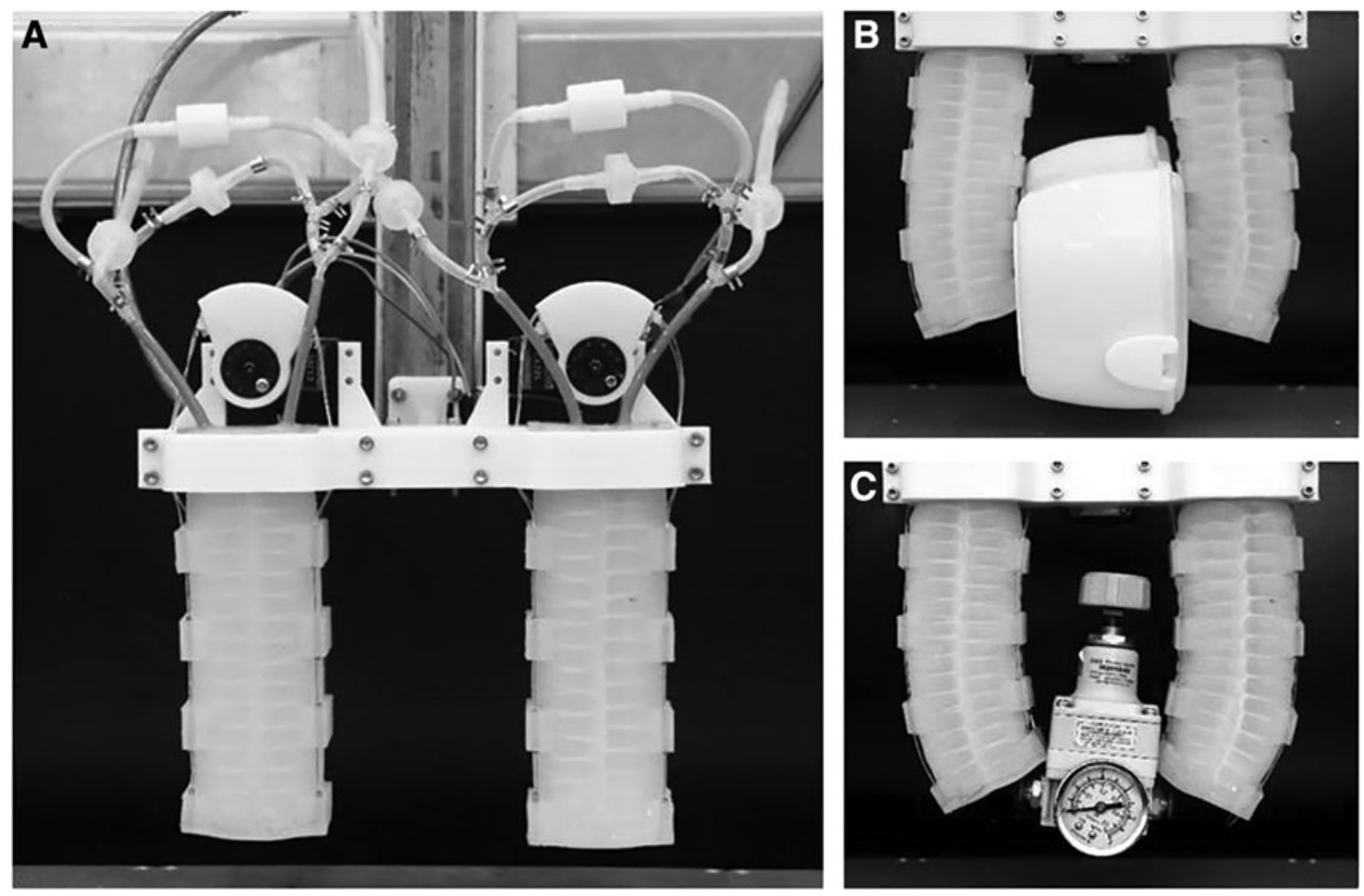

FIG. 6. Grasping demonstration. (A) The complete prototype gripper system (no need for air supply) composed of two SSPAs. (B) Grasp a plastic box (130 g). (C) Grasp a pressure gauge (384 g). 


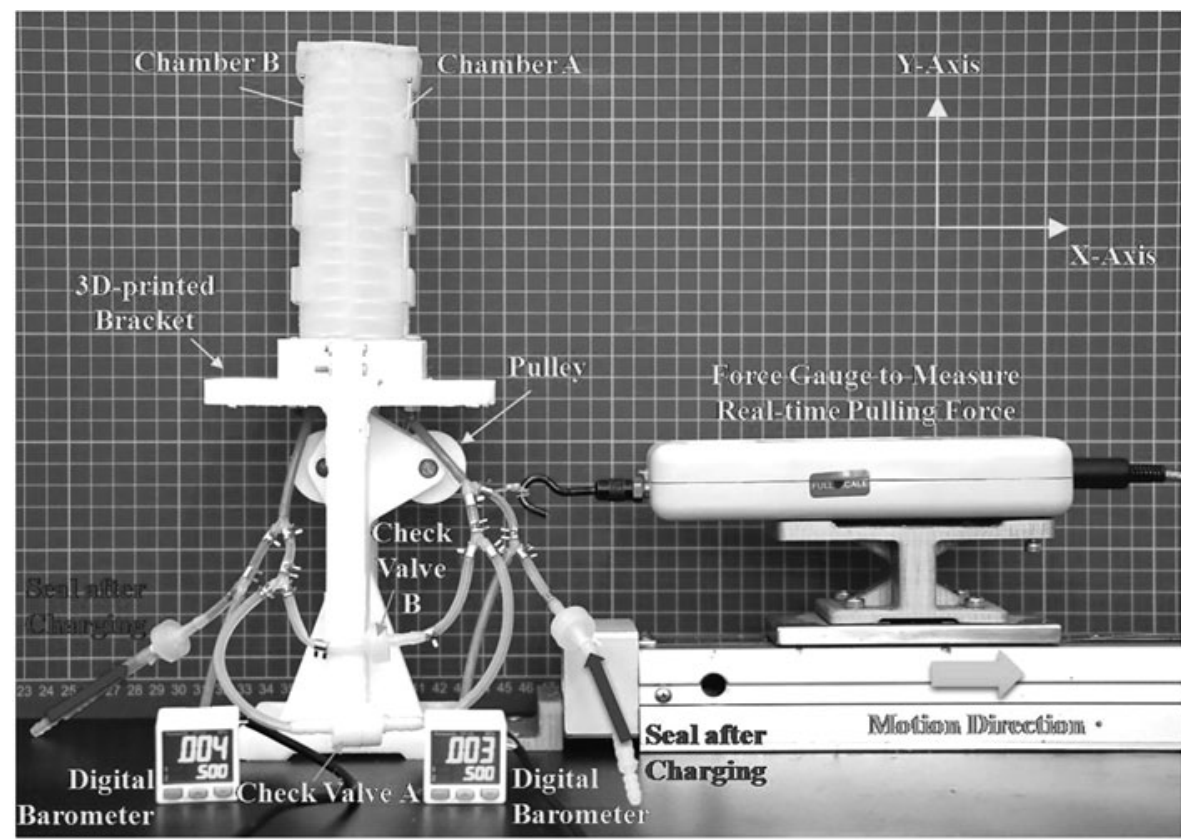

FIG. 7. Experimental setup (pull the tendon along horizontal direction through a pulley). the gripper with two proposed SSPAs, each is controlled by a servo motor. Two grasping demonstrations are shown in Figure $6 \mathrm{~B}$ and C. Since the proposed SSPA has the same design as that of traditional SPAs, the compliance, robustness, and safety of the proposed SSPA are also comparable with those of SPA. The following shows an experimental study of unique characteristics of the proposed actuator.

\section{Experiments and Result Analysis}

\section{Experiments}

To demonstrate the positive effect of air transmission on actuator performance, four groups of experiments were conducted. The experimental setup is shown in Figure 7. The linear drive was placed horizontally along the $\mathrm{X}$-axis. A force gauge (ZHIQU DS2-50N type) was placed on the linear drive. The proposed actuator was installed to a 3D-printed bracket. The tendon was wound around a pulley on the bracket and connected to the force gauge. As for the bending angle measurement, it is calculated offline using video images captured during the bending process. To measure the air pressure change, each chamber A and chamber B was connected to a digital barometer. All the experiments were conducted three times at room temperature. Average data values are used for tabulation and graphical charting.

The first group of experiments is to investigate the effect of differential air pressure on the actuation performance of our proposed SSPA. The check valve A as shown in Figure $2 \mathrm{C}$ installed in the prototype has the cracking pressure of $\Delta P=15 \mathrm{kPa}$. Check valve $\mathrm{B}$ is removed in these experiments. The chamber pressure $P$ is tested at two different values 0 and $10 \mathrm{kPa}$; for each $p$ value, the pressure difference (chamber B larger than chamber A) is set to $0,5,10$, and $15 \mathrm{kPa}$, respectively. The initial pulling force is defined as the force when the tendon is pulled a distance of $10 \mathrm{~mm}$.

The second set of experiments compares the different performance of BSPA and SSPA. The third set of experiments studies the effects of dimension and material on the performance of SSPA. In these experiments, the force gauge on the $\mathrm{X}$-axis pulls the tendon to the right side, while the pulling force is recorded in real time. To ensure the actuator is actuated under the status of quasi-static, the pulling motion is controlled at the slow speed of $0.35 \mathrm{~mm} / \mathrm{s}$. The tendon is pulled a distance of $60 \mathrm{~mm}$. As for the bending angle, it is calculated offline based on captured video images by a camera.

The fourth group of experiments studies the axial stiffness change of the SSPA at different precharged air pressures.

\section{Results and analysis}

The effect of differential pressure value. The results of the first group of experiments are presented in Table 1. It can be seen that the larger the differential pressure, the smaller the initial pulling force to initiate bending. For chamber pressure at $0 \mathrm{kPa}$, the data show that the initial bending force can be reduced by $16 \%$ if the differential pressure is $15 \mathrm{kPa}$. However, when the initial precharged pressure is increased, say at $10 \mathrm{kPa}$, the force reduction is less obvious because a large precharged air pressure means a larger actuator stiffness. A small pressure difference does not influence too much on the initial bending force. However, the fact that increasing the

Table 1. Comparison of Initial Pulling Force at Different Chamber Pressures

\begin{tabular}{lcc}
\hline $\begin{array}{l}\text { Chamber } A \\
\text { pressure }(\mathrm{kPa})\end{array}$ & $\begin{array}{c}\text { Chambers' pressure } \\
\text { difference }(\mathrm{kPa})\end{array}$ & $\begin{array}{c}\text { Initial pulling } \\
\text { force }(\mathrm{N})\end{array}$ \\
\hline 0 & 0 & 0.90 \\
& 5 & 0.88 \\
& 10 & 0.83 \\
10 & 15 & 0.76 \\
& 0 & 1.38 \\
& 5 & 1.35 \\
& 10 & 1.30 \\
& 15 & 1.24 \\
\hline
\end{tabular}


differential pressure will reduce the initial pulling force is always true.

Comparison of BSPA and SSPA. For both BSPA and SSPA, with the increase of pulling force, the actuator bending angle also increases, in an approximately linear relationship as shown in Figure 8A and B. A small pulling force fluctuation might have been caused by friction between the tendon and the pulley, the silicone pad, etc. For both SSPA and BSPA at precharged air pressure $0 \mathrm{kPa}$, the pulling force stops increasing linearly from the actuator bending angle $\sim 40^{\circ}-50^{\circ}$. This might be the result of small stiffness of the actuators at $0 \mathrm{kPa}$ precharged air pressure. With a small stiffness, a smaller pulling force will cause the actuators to buckle. At the buckled state, only a small pulling force will result in a large bending angle. The experiments have also shown that both the SSPA and BSPA can bend up to $100^{\circ}$ (bending angle is defined in Fig. 3B).

When precharged at the same air pressure and actuated to the same bending angle, the SSPA requires a smaller pulling force than the BSPA as shown in Figure 8C. The larger the precharged air pressure, the larger the pulling force difference.
Figure 8D presents the comparison of cumulative work done for SSPA and BSPA when they are both actuated to a bending angle of $80^{\circ}$ at a tendon pulling speed of $0.35 \mathrm{~mm} / \mathrm{s}$. It can be seen that the proposed SSPA has a clear energy saving advantage at all precharged air pressures. Increasing the precharge pressure will increase the energy saving percentage. In the experiments with the prototype SSPA, the energy saving has reached $45 \%$ at the precharged air pressure of $60 \mathrm{kPa}$ when the actuator is actuated to a bending angle of $80^{\circ}$. With this effect, servo motors with small power can be used to drive the proposed actuator, thus reducing the overall weight and size of the proposed actuator.

Theoretic modeling of actuator chamber air pressure change against bending angle has been validated by experimental results as shown in Figure 8E. For the proposed SSPA, air pressure in the two chambers $\left(\mathrm{P}_{\mathrm{A}-\mathrm{AB}}\right)$ should be equalized and remains flat regardless of the bending angle. In the actual bending tests, the two chambers have the same pressure $\left(\mathrm{P}_{\mathrm{E}-\mathrm{AB}}\right)$, yet the pressure is on a slowly increasing trend. This may have been because the overall chamber volume was slowly reduced in the bending process. Nevertheless, the difference is marginal. For the BSPA, the experimental data $\left(\mathrm{P}_{\mathrm{E}-\mathrm{A}^{\prime}}\right.$ and $\left.\mathrm{P}_{\mathrm{E}-\mathrm{B}^{\prime}}\right)$
A

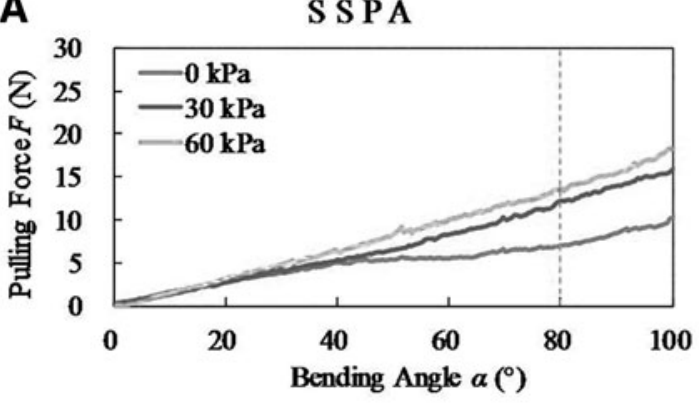

C

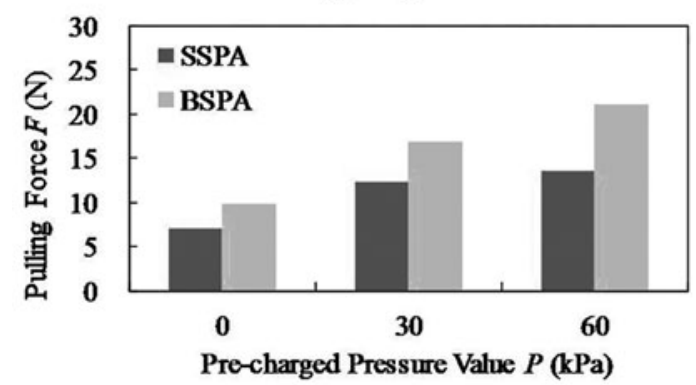

B
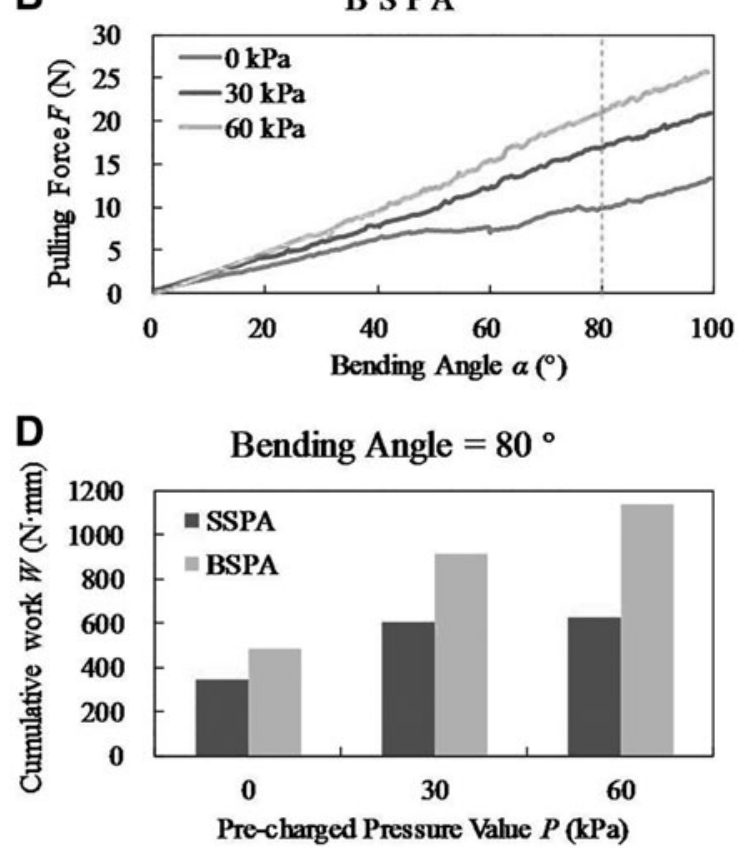

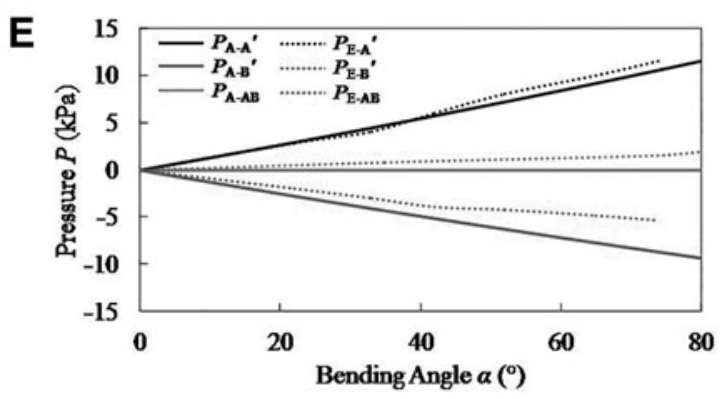

FIG. 8. Experimental results of SSPA and BSPA. (A) Pulling force versus bending angle for the proposed SSPA. (B) Pulling force versus bending angle for BSPA. (C) Pulling force comparison (at bending angle $80^{\circ}$ ) for SSPA and BSPA with different precharged pressures. (D) Comparison of cumulative work done between the two types of actuators when the bending angle reaches $80^{\circ}$. (E) Chamber pressure versus bending angle. 
A
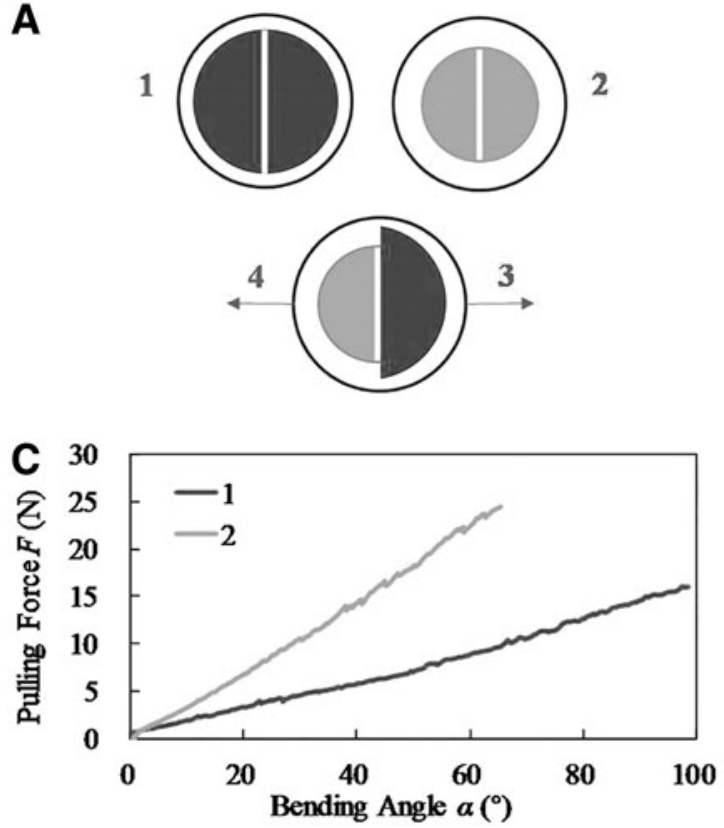
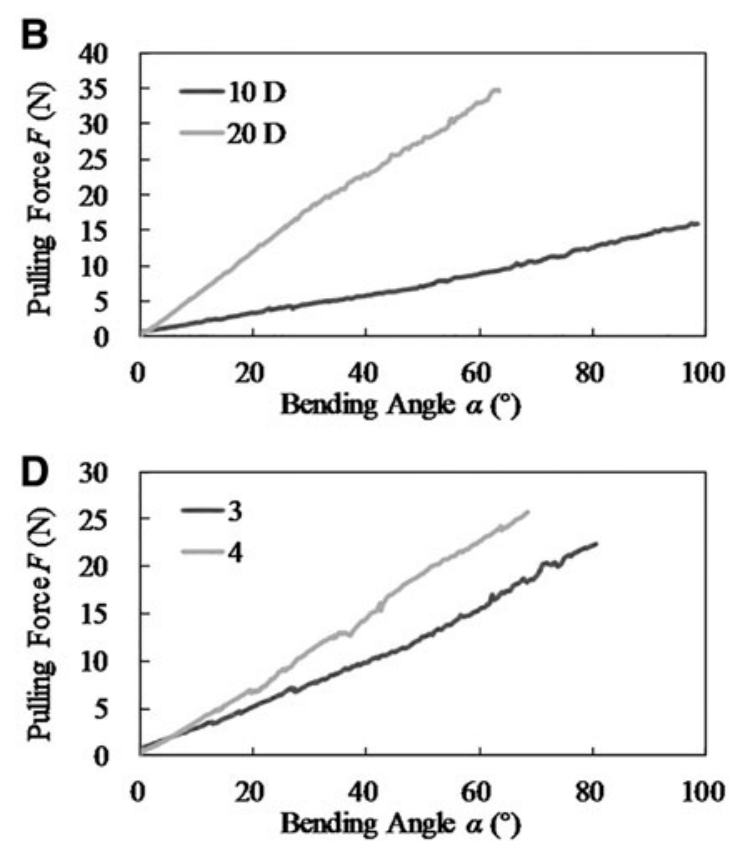

FIG. 9. Effects of different chamber sizes and materials. (A) Chamber sizes: A1, two chambers with both radii at $16 \mathrm{~mm}$, outer radius at $20 \mathrm{~mm}$; $\mathrm{A} 2$, two chambers with both radii at $12 \mathrm{~mm}$, outer radius at $20 \mathrm{~mm}$; A3, different inner chamber size, the larger chamber with inner radius $16 \mathrm{~mm}$, and smaller chamber with inner radius $12 \mathrm{~mm}$, outer radius at $20 \mathrm{~mm}$. (B) Two A1 made of silicone rubber with hardness of 10D and 20D. (C) A1 and A2 made of 10D silicone rubber. (D) A3 made of 10D silicone rubber bent to direction 3 and 4.

are generally in agreement with the data from theoretic modeling $\left(\mathrm{P}_{\mathrm{A}-\mathrm{A}^{\prime}}\right.$ and $\left.\mathrm{P}_{\mathrm{A}-\mathrm{B}^{\prime}}\right)$.

The effects of size and material. To obtain the effects of size and material property on the performance of the proposed SSPAs, an experiment is designed as illustrated in Figure 9A. The outer radii of all sample actuators are the same at $20 \mathrm{~mm}$. All the experiments were conducted at precharged air pressure of $0 \mathrm{kPa}$. At this air pressure, the effect of size and material could be maximized.
In Figure 9B, the pulling force required by the actuator made of 10D hardness silicone rubber is much smaller than that of 20D silicone rubber under the same bending angle. Thus, the actuator made of 20D hardness silicone rubber has a much larger self-stiffness $\left(35 / 8=4.38\right.$ measured at $60^{\circ}$ bending angle), around 4.35 times of the one made of $10 \mathrm{D}$ silicone rubber.

In Figure 9C, the effect of chamber size on actuator stiffness is studied. It can be seen that the pulling force required by the actuator with larger chambers is smaller than that with

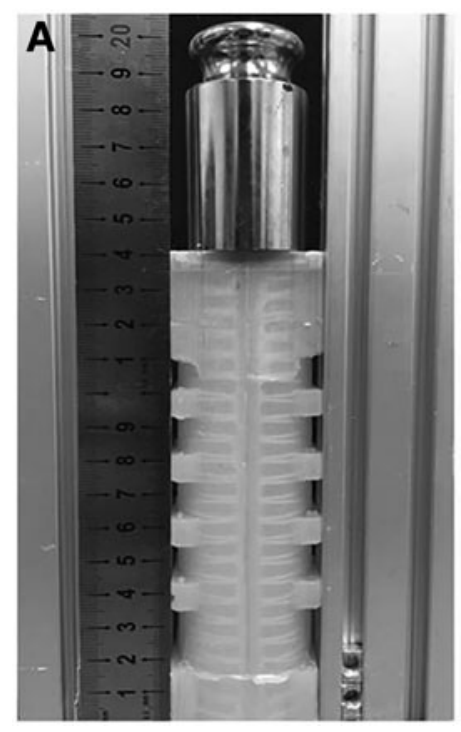

B

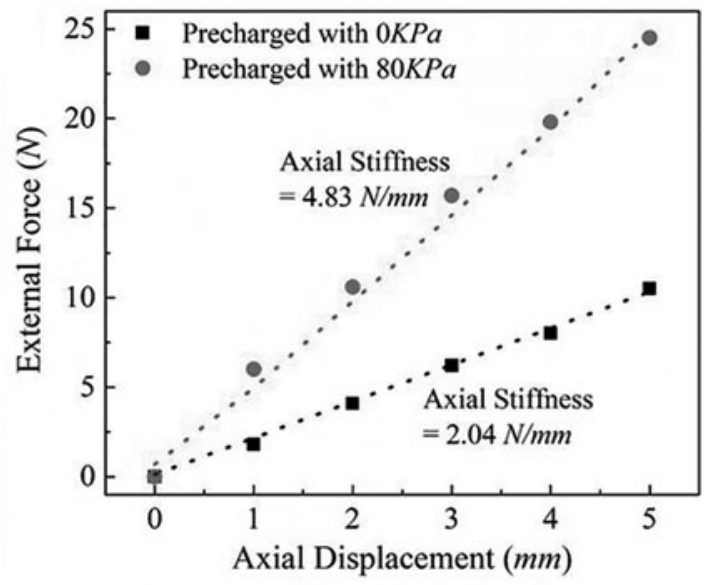

FIG. 10. Axial stiffness for the proposed soft self-pumping actuator. (A) The experimental setup. (B) Stiffness at two different precharged chamber pressures. 
smaller chamber size at the same bending angle. Thus, the actuator composed of two smaller chambers has a larger stiffness.

An interesting test result is illustrated in Figure 9D, where the actuator has a large chamber and a small chamber (shown in Fig. 9A). A smaller force is required to bend toward the side of a larger chamber compared with bending toward the side of a smaller chamber when the bending angle is the same. The primary reason might be that the wall thickness of the bigger chamber is smaller; thus, its stiffness is smaller too. This observation is useful in designing the proposed SSPAs whose bending is primarily toward one side.

Axial stiffness. This set of experiments is to investigate the stiffness change of our proposed SSPA at different precharged air pressures. Sun et al. ${ }^{25}$ have shown that stiffness of SPAs changes as actuation air pressure changes. In this study, the axial stiffness of the proposed actuator was studied. In this research, the axial stiffness is calculated by using the applied weight divided by the axial displacement as shown in the experimental setup in Figure 10A. Figure 10B illustrates the relationship between the added weight and the axial displacement. It is very close to a linear relationship. Thus, the data are fitted to a straight line whose slope is the axial stiffness of the prototype actuator. In the figure, the calculated axial stiffness of the prototype SSPA with a $80 \mathrm{kPa}$ precharged air pressure is $4.83 \mathrm{~N} / \mathrm{mm}$, which is more than twofold compared with the axial stiffness of $2.04 \mathrm{~N} / \mathrm{m}$ at $0 \mathrm{kPa}$ precharged air pressure. The experimental result illustrates that the same physical SSPA may have different initial stiffness depending on the precharged air pressure. It is also possible to regulate the air pressure (thus the stiffness of the actuator) by simultaneously contracting tendons on both sides. This effect will be further studied by the authors.

\section{Conclusion}

To solve problems resulted from insufficient energy in soft robotics, previous research mainly focused on developing new energy sources or devices with larger energy capacities. In this study, a self-pumping concept has been proposed to reduce the required force in bending actuation. Modeling and experimental studies for a sample self-pumping soft actuator have been conducted. Results have shown clear force reduction and energy saving using the proposed concept. The contributions of the proposed design are summarized: (1) the proposed SSPA is the first attempt for TSA design with selfpumping features aimed at actuation force reduction, thus saving operation energy; (2) the check valves between the air chambers can balance the pressure differences and transmit energy to help actuation when the actuator is being bent; (3) the results prove that the differential pressures of the proposed actuator require less force to initiate bending; (4) the experiments have proved that the proposed SSPA requires less force and energy for actuation compared with the BSPA; (5) the same SSPA may have different initial stiffness by precharging it to different air pressures; and (6) the SSPA does not need any pneumatic control systems for its operation, thus it can be used for untethered applications. Even though this work is only a preliminary study, it is believed that more future soft actuator designs will utilize the proposed self-pumping concept.

\section{Author Disclosure Statement}

No competing financial interests exist.

\section{Funding Information}

This work was supported by National Natural Science Foundation of China (NSFC) under Project 51805443.

\section{References}

1. Rafsanjani A, Zhang Y, Liu B, et al. Kirigami skins make a simple soft actuator crawl. Sci Robot 2018;3:eaar7555.

2. Ghosh S, Ghosh A. Mobile nanotweezers for active colloidal manipulation. Sci Robot 2018;3:eaaq0076.

3. Yang Y, Chen YH, Li Y, et al. Bioinspired robotic fingers based on pneumatic actuator and 3D printing of smart material. Soft Robot 2017;4:147-162.

4. Banerjee H, Ren H. Optimizing double-network hydrogel for biomedical soft robots. Soft Robot 2017;4:191-201.

5. Li Y, Chen YH, Yang Y, et al. Passive particle jamming and its stiffening of soft robotic grippers. IEEE Trans Robot 2017;33:446-455.

6. Terryn S, Brancart J, Lefeber D, et al. Self-healing soft pneumatic robots. Sci Robot 2017;2:eaan4268.

7. Robertson MA, Paik J. New soft robots really suck: vacuum-powered systems empower diverse capabilities. Sci Robot 2017;2: eaan6357.

8. Kurumaya S, Phillips BT, Becker KP, et al. A modular soft robotic wrist for underwater manipulation. Soft Robot 2018;5:399-409.

9. Waynelovich J, Frey T, Baljon A, et al. Versatile and dexterous soft robotic leg system for untethered operations. Soft Robot 2016;3:64-70.

10. Kofod G, Wirges W, Paajanen M, et al. Energy minimization for self-organized structure formation and actuation. Appl Phys Lett 2007;90:081916.

11. Desbiens AB, Bigué JPL, Véronneau C, et al. On the potential of hydrogen-powered hydraulic pumps for soft robotics. Soft Robot 2017;4:367-378.

12. Boyvat M, Koh JS, Wood RJ. Addressable wireless actuation for multijoint folding robots and devices. Sci Robot 2017;2:eaan1544.

13. Nemitz MP, Mihaylov $\mathrm{P}$, Barraclough TW, et al. Using voice coils to actuate modular soft robots: wormbot, an example. Soft Robot 2016;3:198-204.

14. Yang GZ, Bellingham J, Dupont PE, et al. The grand challenges of science robotics. Sci Robot 2018;3:eaar7650.

15. Shui L, Zhu L, Yang Z, et al. Energy efficiency of mobile soft robots. Soft Matter 2017;13:8223-8233.

16. Marchese AD, Onal CD, Rus D. Soft robot actuators using energy-efficient valves controlled by electropermanent magnets. Proceedings of the 2011 IEEE/RSJ International Conference on Intelligent Robots and Systems (IROS), San Francisco, CA, September 25-30, 2011, pp. 4446-4445.

17. Wei Y, Chen YH, Ren T, et al. A novel, variable stiffness robotic gripper based on integrated soft actuating and particle jamming. Soft Robot 2016;3:134-143.

18. Trolley MT, Shepherd RF, Mosadegh B, et al. A resilient, untethered soft robot. Soft Robot 2014;1:213-223.

19. Kobayashi H, Hyodo K, Ogane D. On tendon-driven robotic mechanisms with redundant tendons. Int J Robot Res 1998;17:561-571.

20. Shirafuji S, Ikemoto S, Hosoda K. Development of a tendon-driven robotic finger for an anthropomorphic robotic hand. Int J Robot Res 2014;33:677-693. 
21. Renda F, Giorelli M, Calisti M, et al. Dynamic model of a multibending soft robot arm driven by cables. IEEE Trans Robot 2014;30:1109-1122.

22. Li Y, Chen YH, Ren T, et al. Passive and active particle damping in soft robotic actuators. 2018 IEEE International Conference on Robotics and Automation (ICRA), May 21-25, 2018, Brisbane, Australia, pp. 15471542.

23. Li Y, Chen YH, Ren T, et al. Precharged pneumatic soft actuators and their applications to untethered soft robots. Soft Robot 2018;5:567-575.

24. Polygerinos P, Wang Z, Overvelde JT, et al. Modeling of soft fiber-reinforced bending actuators. IEEE Trans Robot 2015;31:778-789.
25. Sun Y, Song YS, Paik J. Characterization of silicone rubber based soft pneumatic actuators. 2013 IEEE/RSJ International Conference on Intelligent Robots and Systems (IROS), 2013, Tokyo, Japan, pp. 4446-4453.

Address correspondence to: Yonghua Chen The Department of Mechanical Engineering The University of Hong Kong Pokfulam Road 999077 Hong Kong China

E-mail: yhchen@hku.hk 